\title{
Microwave spectroscopy and characterization of the helical conformer of perfluorohexane
}

\author{
Joseph A. Fournier, Congtin L. Phan, and Robert K. Bohn* \\ Department of Chemistry, University of Connecticut, Storrs, CT, 06269-3060, USA \\ E-mail: Robert.bohn@uconn.edu
}

This paper is dedicated to William F. Bailey in honor of his $65^{\text {th }}$ birthday

\begin{abstract}
The lowest energy conformer of perfluorohexane $\left(\mathrm{C}_{6} \mathrm{~F}_{14}\right)$ is helical and its microwave rotational spectrum has been observed and assigned. The helicity along the carbon chain results in overall $C_{2}$ symmetry with the $C_{2}$ axis corresponding to the c-principal axis. The assignment of a c-type spectrum confirms the structure of $\mathrm{C}_{6} \mathrm{~F}_{14}$ to be helical. The rotational constants are $A$ 824.9001(9) $\mathrm{MHz}, B$ 202.2195(8) MHz, and C 198.3355(10) MHz. The molecular parameters were characterized by scaling computed models by the square root of the ratio of the observed second moments to those computed. The scaled structures exactly reproduce the observed second moments. From this scaling approach, the exterior $\mathrm{C}_{1} \mathrm{C}_{2} \mathrm{C}_{3} \mathrm{C}_{4}$ dihedral angle is $16.7^{\circ}$ and the interior $\mathrm{C}_{2} \mathrm{C}_{3} \mathrm{C}_{4} \mathrm{C}_{5}$ dihedral angle is $18.0^{\circ}$ away from trans. Computed models at the PBE0/VTZ level of theory are in excellent agreement with the experimental results.
\end{abstract}

Keywords: Perfluorohexane, tetradecafluorohexane, molecular structure, microwave spectroscopy

\section{Introduction}

The polymer polytetrafluoroethylene (PTFE) is well characterized and known to have a helical structure by X-ray diffraction studies on single fibers. ${ }^{1}$ A helix can be described by either a helical perspective or a molecular perspective. In the helical perspective, the helix is defined by the following three parameters: the distance from each atom to the helical axis (the helical radius), $\rho$; the helical angle about the helical axis, $\theta$; and the translation from one atom to the next (pitch) along the helical axis, $\mathrm{d}$. In the molecular perspective, the helix is defined as follows: the $\mathrm{C}-\mathrm{C}$ bond length, $\mathrm{r}$; the CCC bond angle, $\varphi$; and the CCCC dihedral angle, $\tau$. A visual representation of the two perspectives is displayed in Figure 1. The mathematical relationships for converting between the two perspectives are given in Equations 1 and 2., ${ }^{2,3}$ For the low 
temperature $\left(<19^{\circ} \mathrm{C}\right)$ phase II form of PTFE, the helical angle is periodic and equal to $13.8^{\circ} .4$ This corresponds to a CCCC dihedral angle of about $17^{\circ}$ away from trans. The helicity of PTFE is attributed to steric and dipole repulsions of $\mathrm{F}$ atoms on alternate carbons. A small helical twist along the carbon chain helps alleviate these repulsions (Figure 2).
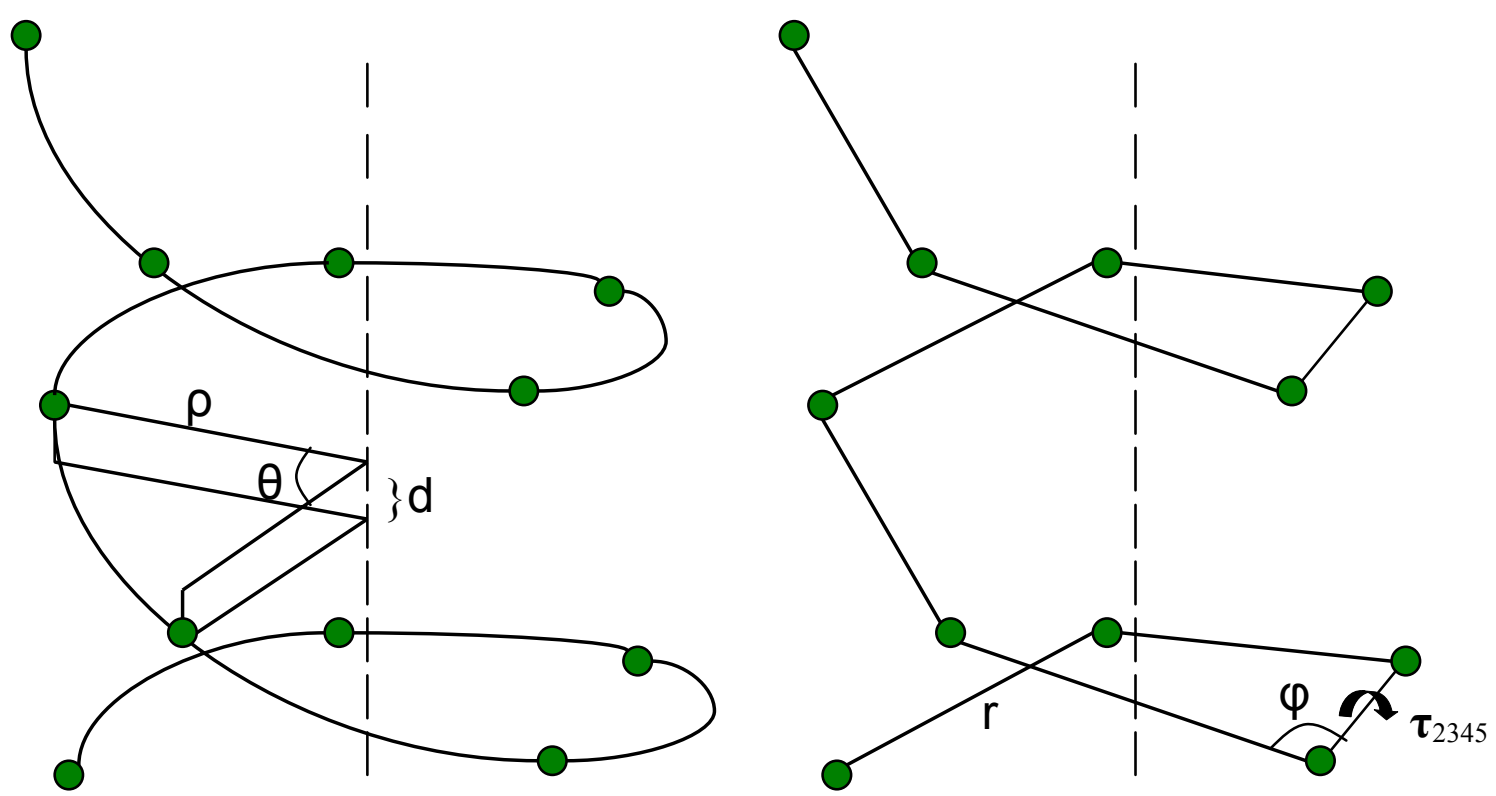

Figure 1. Helical (left) and molecular (right) perspectives for describing a helix. The dashed line represents the helical axis and the green circles represent $\mathrm{CF}_{2}$ groups.

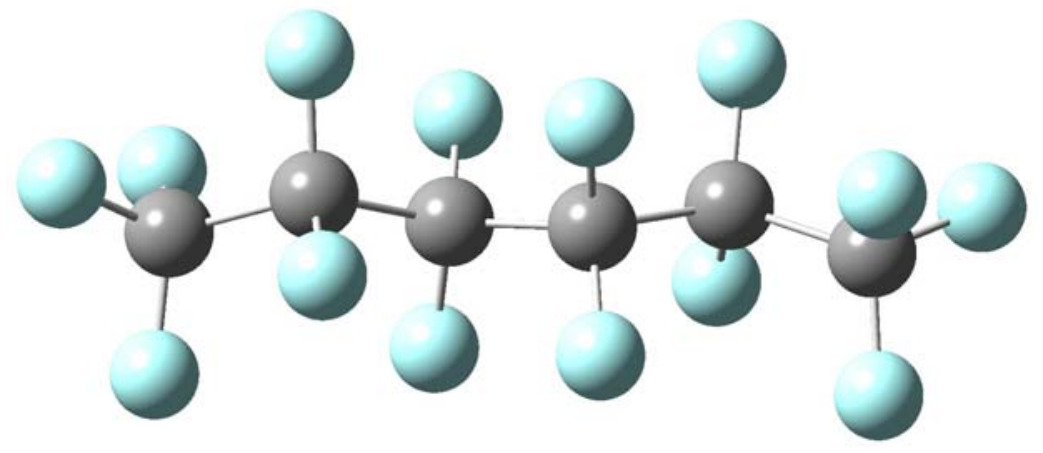

Figure 2. Top view of $\mathrm{C}_{6} \mathrm{~F}_{14}$.

Experimental and computational studies on smaller perfluoroalkane oligomers also show the lowest energy structures to be helical, beginning with perfluorobutane $\left(\mathrm{C}_{4} \mathrm{~F}_{10}\right){ }^{5-15}$ The helical (trans), gauche, and ortho $\left(\tau \sim 90^{\circ}\right)$ conformers of $\mathrm{C}_{4} \mathrm{~F}_{10}$ were observed by nitrogen matrixisolated IR spectroscopy. ${ }^{8}$ Only the gauche form was observed in a microwave study, ${ }^{16}$ with the helical form apparently having too small a dipole moment to be observable and the ortho form 
relaxing to the lower energy gauche form. ${ }^{17}$ The $\mathrm{CCCC}$ dihedral angle of $\mathrm{C}_{4} \mathrm{~F}_{10}$ has not been determined experimentally and computations predict a twist of $\sim 14^{\circ}$ from trans. ${ }^{6-13}$

$$
\begin{aligned}
& \cos \theta=1 / 2(-\cos \varphi+\cos \tau-\cos \varphi \cos \tau-1) \\
& d^{2}=r^{2}(1-\cos \tau)(1-\cos \varphi) /(3+\cos \varphi-\cos \tau+\cos \varphi \cos \tau) \\
& \rho^{2}=2 r^{2}(1+\cos \varphi) /(3+\cos \varphi-\cos \tau+\cos \varphi \cos \tau)^{2} \\
& r^{2}=d^{2}+4 \rho^{2} \sin ^{2}(\theta / 2) \\
& \cos (\varphi / 2)=\left(1-d^{2} / r^{2}\right)^{1 / 2} \sin (\theta / 2) \\
& \tan (\tau / 2)=(d / r) \tan (\theta / 2)
\end{aligned}
$$

The microwave spectra of perfluoropentane $\left(\mathrm{C}_{5} \mathrm{~F}_{12}\right)$ and its three ${ }^{13} \mathrm{C}$ isotopomers have recently been assigned. ${ }^{15}$ The value of the dihedral angle was determined by scaling computed models to exactly reproduce the observed second moments. The CCCC dihedral angle was calculated to be $17^{\circ} \pm 1^{\circ}$ from trans using this method, in good agreement with previous computational studies. ${ }^{7,9-14}$ In the same study, the structure of perfluoropropane $\left(\mathrm{C}_{3} \mathrm{~F}_{8}\right)$ was found to be non-helical $\left(C_{2 \mathrm{v}}\right)$. Apparently, the steric and dipole interactions in the three carbon chain are not sufficient to cause a twist in the structure.

To continue characterization of short perfluoroalkanes, a microwave spectroscopic study on perfluorohexane $\left(\mathrm{C}_{6} \mathrm{~F}_{14}\right)$ was performed. The two CCCC dihedral angles in $\mathrm{C}_{6} \mathrm{~F}_{14}$ are predicted to be about $16^{\circ}-18^{\circ}$ away from trans by various computational methods. ${ }^{7,11,12,14} \mathrm{C}_{6} \mathrm{~F}_{14}$, if helical, would have a dipole moment and, therefore, be observable by microwave spectroscopy. The microwave spectrum of the lowest energy helical conformer of $\mathrm{C}_{6} \mathrm{~F}_{14}$ (Figures 2 and 3) has been observed and assigned and the molecular geometry analyzed.
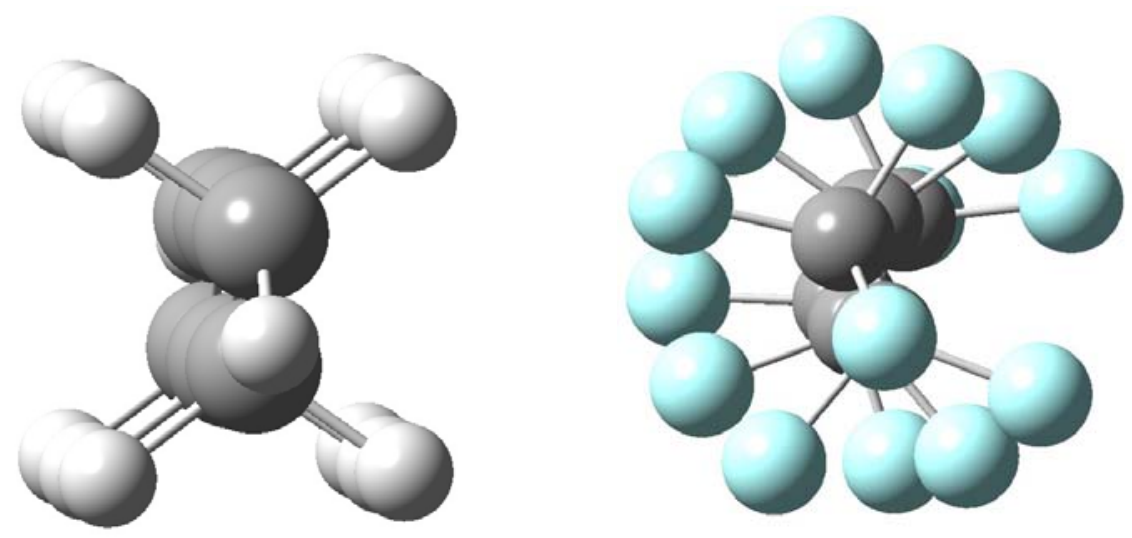

Figure 3. The $C_{2 \mathrm{~h}}$ structure of $\mathrm{C}_{6} \mathrm{H}_{14}$ with dihedral angles of $180^{\circ}$ (left) and the $C_{2}$ structure of $\mathrm{C}_{6} \mathrm{~F}_{14}$ with dihedral angles of $\sim 17^{\circ}$ from trans (right). 


\section{Results and Discussion}

The lowest energy all trans conformer of n-hexane $\left(C_{2 \mathrm{~h}}\right)$ lacks a dipole moment and is therefore spectroscopically unobservable. The lowest energy all trans structure of perfluoroalkanes, beginning with $\mathrm{C}_{4} \mathrm{~F}_{10}$, is helical. A figure of the helical structure of $\mathrm{C}_{6} \mathrm{~F}_{14}$, similar to Figure 3, given in reference 14 sparked our interest as this structure clearly has a dipole moment. The helicity of perfluoroalkanes results in structures with overall $C_{2}$ symmetry. For an odd numbered chain, like $\mathrm{C}_{5} \mathrm{~F}_{12}$, ${ }^{15}$ the $C_{2}$ axis corresponds to the b-axis and b-type transitions are observed. For an even numbered chain the $C_{2}$ axis becomes the c-axis and, thus, c-type transitions are expected for $\mathrm{C}_{6} \mathrm{~F}_{14}$. The observation and assignment of the microwave spectrum of $\mathrm{C}_{6} \mathrm{~F}_{14}$ to c-type transitions (see Table 1 and Supplementary Table 1) unambiguously shows that the lowest energy structure of $\mathrm{C}_{6} \mathrm{~F}_{14}$ is helical. The observation of c-type transitions requires that the molecule have a non-zero dipole moment along the $\mathrm{c}$ axis. If the molecule were non-helical, the structure would have $\mathrm{C}_{2 \mathrm{~h}}$ symmetry and no dipole moment along any principal axis.

Table 1. Spectroscopic constants of $\mathrm{C}_{6} \mathrm{~F}_{14}$

\begin{tabular}{cc}
\hline & $\mathrm{C}_{6} \mathrm{~F}_{14}$ \\
\hline $\mathrm{A} / \mathrm{MHz}$ & $824.9001(9)$ \\
$\mathrm{B} / \mathrm{MHz}$ & $202.2195(8)$ \\
$\mathrm{C} / \mathrm{MHz}$ & $198.3355(10)$ \\
$\mathrm{D}_{\mathrm{J}} / \mathrm{kHz}$ & $0.00165(13)$ \\
$\mathrm{P}_{\mathrm{aa}} / \mathrm{u} \AA^{2}$ & 2217.304 \\
$\mathrm{P}_{\mathrm{bb}} / \mathrm{u} \AA^{2}$ & 330.798 \\
$\mathrm{P}_{\mathrm{cd}} / \mathrm{u} \AA^{2}$ & 281.857 \\
$\mathrm{Kappa}$ & -0.9876022 \\
No. Lines & 46 \\
Std. Dev./kHz & 1.8 \\
\hline
\end{tabular}

The structure of $\mathrm{C}_{3} \mathrm{~F}_{8}$ was recently determined to be non-helical $\left(C_{2 \mathrm{v}}\right){ }^{15}$ The $\mathrm{P}_{\mathrm{cc}}$ second moment is $134.338 \mathrm{u} \AA^{2}$, or $44.78 \mathrm{u} \AA^{2}$ per $\mathrm{CF}_{2} / \mathrm{CF}_{3}$ group. Assuming transferability, $\mathrm{C}_{6} \mathrm{~F}_{14}$ would have a $\mathrm{P}_{\mathrm{cc}}$ of 6 x 44.78 or $268.68 \mathrm{u} \AA^{2}$ if it exhibited $C_{2 \mathrm{~h}}$ symmetry. The observed $\mathrm{P}_{\mathrm{cc}}$ is $281.857 \mathrm{u} \AA^{2}$, or $46.98 \mathrm{u} \AA^{2}$ per $\mathrm{CF}_{2} / \mathrm{CF}_{3}$ group. This increase in $\mathrm{P}_{\mathrm{cc}}$ also confirms the helical structure of $\mathrm{C}_{6} \mathrm{~F}_{14}$ because $\mathrm{C}$ atoms as well as $\mathrm{F}$ atoms lie outside the ab plane and contribute to $\mathrm{P}_{\mathrm{cc}}$. The $\mathrm{P}_{\mathrm{cc}}$ second moment increases steadily as the $\mathrm{C}$ chain becomes longer. The $\mathrm{P}_{\mathrm{cc}}$ per $\mathrm{CF}_{2} / \mathrm{CF}_{3}$ group for $\mathrm{C}_{3} \mathrm{~F}_{8}{ }^{15}$ is $44.78 \mathrm{u} \AA^{2}$ and is predicted to be $45.16 \mathrm{u} \AA^{2}$ for $\mathrm{C}_{4} \mathrm{~F}_{10}$ (from PBE0/VTZ calculations). This value increases to $45.92 \mathrm{u} \AA^{2}$ for $\mathrm{C}_{5} \mathrm{~F}_{12}{ }^{15}$ and to $46.98 \mathrm{u} \AA^{2}$ for $\mathrm{C}_{6} \mathrm{~F}_{14}$. The trend demonstrates that as the $\mathrm{C}$ chain length increases more mass is displaced outside the ab plane and contributes to $\mathrm{P}_{\mathrm{cc}}$ due to the helicity of the structures.

The microwave data alone do not allow for an exact determination of the CCCC dihedral angles. To analyze the structural parameters of $\mathrm{C}_{6} \mathrm{~F}_{14}$, the principal coordinates of the computed 
model were scaled by the square root of the ratio of the observed second moments to the computed second moments. This shifts the coordinates of each atom to exactly reproduce the observed second moments. Computations were performed at the PBE0/VTZ level of theory using the Gaussian 03 program. ${ }^{18}$ The PBE0 functional has been shown to accurately model perfluoroalkanes $^{13,15}$ and is again evident by the excellent agreement between the observed and computed spectroscopic constants for $\mathrm{C}_{6} \mathrm{~F}_{14}$ (Table 2). The scale factors are 0.99996 for the acoordinates, 1.00167 for the b-coordinates, and 1.00288 for the c-coordinates. Since the scale factors are nearly equal to 1 , the structural parameters do not change much from the computed model (Table 3). The overall $C_{2}$ symmetry of $\mathrm{C}_{6} \mathrm{~F}_{14}$ allows for two distinct dihedral angles. The exterior $\mathrm{C}_{1} \mathrm{C}_{2} \mathrm{C}_{3} \mathrm{C}_{4}$ dihedral angle (also equal to $\mathrm{C}_{3} \mathrm{C}_{4} \mathrm{C}_{5} \mathrm{C}_{6}$ ) is $16.7^{\circ}$ away from trans and the interior $\mathrm{C}_{2} \mathrm{C}_{3} \mathrm{C}_{4} \mathrm{C}_{5}$ dihedral angle is $18.0^{\circ}$ away from trans using this scaling approach. The exterior dihedral angle is in agreement with the single dihedral angle of about $17^{\circ}$ from trans determined in $\mathrm{C}_{5} \mathrm{~F}_{12}$ using this same scaling approach. ${ }^{15}$ The interior dihedral angle is slightly larger than the exterior dihedral angle likely because the interior of the molecule is more sterically crowded.

Table 2. Comparison of observed spectroscopic constants to the computed and scaled models

\begin{tabular}{|c|c|c|c|}
\hline & Obs'd & PBE0/VTZ & $\begin{array}{c}\text { Scaled } \\
\text { PBE0/VTZ }\end{array}$ \\
\hline $\mathrm{A} / \mathrm{MHz}$ & 824.9001 & 828.6 & 824.90 \\
\hline $\mathrm{B} / \mathrm{MHz}$ & 202.2195 & 202.3 & 202.22 \\
\hline $\mathrm{C} / \mathrm{MHz}$ & 198.3355 & 198.4 & 198.34 \\
\hline $\mathrm{P}_{\mathrm{aa}} / \mathrm{u} \AA^{2}$ & 2217.304 & 2217.5 & 2217.30 \\
\hline $\mathrm{P}_{\mathrm{bb}} / \mathrm{u} \AA^{2}$ & 330.798 & 329.7 & 330.80 \\
\hline$P_{c c} / u \AA^{2}$ & 281.857 & 280.2 & 281.86 \\
\hline
\end{tabular}

Table 3. Selected structural parameters from the computed and scaled models

\begin{tabular}{ccc}
\hline & PBE0/NTZ & Scaled PBE0/VTZ \\
\hline $\mathrm{C}_{1}-\mathrm{C}_{2}$ & 1.555 & 1.556 \\
$\mathrm{C}_{2}-\mathrm{C}_{3}$ & 1.558 & 1.559 \\
$\mathrm{C}_{3}-\mathrm{C}_{4}$ & 1.561 & 1.561 \\
$\underline{I} \mathrm{C}_{1} \mathrm{C}_{2} \mathrm{C}_{3}$ & 114.0 & 113.9 \\
$\underline{I} \mathrm{C}_{3} \mathrm{C}_{4} \mathrm{C}_{5}$ & 113.0 & 112.9 \\
$\mathrm{C}_{1} \mathrm{C}_{2} \mathrm{C}_{3} \mathrm{C}_{4}$ & 16.7 & 16.7 \\
$\mathrm{C}_{2} \mathrm{C}_{3} \mathrm{C}_{4} \mathrm{C}_{5}$ & 18.0 & 18.0 \\
\hline
\end{tabular}

The scaled $\mathrm{C}_{6} \mathrm{~F}_{14}$ structure was converted to helical parameters using Equations 1 and 2 above, averaging bond lengths, bond angles, and the dihedral angles. The helical radius $\rho$ is $0.434 \AA$, the helical angle $\theta$ is $14.3^{\circ}$, and the pitch $\mathrm{d}$ is $1.298 \AA$. These values are in reasonable 
agreement with the $0.42 \AA$ helical radius, $13.8^{\circ}$ helical angle, and $1.292 \AA$ pitch of the low temperature phase II form of PTFE. ${ }^{3}$ By comparison, the determined helical parameters of $\mathrm{C}_{5} \mathrm{~F}_{12}$ from a scaled MP2/VTZ model are $0.431 \AA$ helical radius, $14.1^{\circ}$ helical angle, and $1.297 \AA$ pitch. ${ }^{15}$

\section{Conclusions}

The helical structure of $\mathrm{C}_{6} \mathrm{~F}_{14}$ has been observed by assignment of its c-type rotational spectrum, consistent with overall $C_{2}$ symmetry. The structure was characterized by scaling a PBE0/VTZ computed model to exactly reproduce the observed second moments. The scaled structure has a $\mathrm{C}_{1} \mathrm{C}_{2} \mathrm{C}_{3} \mathrm{C}_{4}$ exterior dihedral angle of $16.7^{\circ}$ from trans and a $\mathrm{C}_{2} \mathrm{C}_{3} \mathrm{C}_{4} \mathrm{C}_{5}$ interior dihedral angle of $18.0^{\circ}$ from trans. In terms of helical parameters $\mathrm{C}_{6} \mathrm{~F}_{14}$ has a helical angle of about $14^{\circ}$, in good agreement with the $13.8^{\circ}$ helical angle of the low temperature phase II form of PTFE.

\section{Experimental Section}

General. $\mathrm{C}_{6} \mathrm{~F}_{14}$ was purchased from Synquest Laboratories and studied directly. Vapor of the sample was transferred to a $7 \mathrm{~L}$ stainless steel tank to a pressure of $0.04 \mathrm{~atm}$. $6.5 \mathrm{~atm} \mathrm{He}$ was added to produce a $0.6 \%$ sample mixture. Pulses of the sample mixture at 1.75 atm were admitted at $5 \mathrm{~Hz}$ into the pulsed-jet Fourier transform microwave spectrometer ${ }^{19}$ of the Southern New England Microwave Consortium. ${ }^{20}$ Five microwave pulses were observed per gas pulse and rotational transitions were measured in the $6-11 \mathrm{GHz}$ range, although the complete spectrum was not scanned. Transitions were observed as Doppler doublets with estimated uncertainties to be about $2 \mathrm{kHz}$. The rotational temperature of the expanded gas is estimated to be about $5 \mathrm{~K}$. Three observed transitions at $7624.38 \mathrm{MHz}, 8024.94 \mathrm{MHz}$, and $8425.50 \mathrm{MHz}$ were tentatively assigned to the $5_{50}-4_{40} / 5_{51}-4_{41}, \quad 6_{51}-5_{41} / 6_{52}-5_{42}$, and $7_{52}-6_{42} / 7_{53}-6_{43}$ degenerate pairs, respectively.This tentative assignment predicted the $8_{53}-7_{43} / 8_{54}-7_{44}$ degenerate transitions to be around $8826 \mathrm{MHz}$. The assignment proved to be correct as a line was observed at $8826.05 \mathrm{MHz}$, and several more of these degenerate pairs were measured. Since the transitions are doubly degenerate, $\mathrm{B}$ and $\mathrm{C}$ are not distinguishable. A host of non-degenerate lines were predicted from the new assignment and were measured to distinguish $\mathrm{B}$ and $\mathrm{C}$. The transitions were fit using rotational constants and one quartic centrifugal distortion constant using Watson's $\mathrm{S}$ reduction ${ }^{21}$ to an rmsd of $1.8 \mathrm{kHz}$. The use of only one centrifugal distortion constant that is quite small indicates that $\mathrm{C}_{6} \mathrm{~F}_{14}$ is very rigid. The observed spectroscopic constants are displayed in Table 1 and the 46 assigned transitions are listed in Supplementary Table 1. 


\section{Acknowledgments}

Dan Frohman, Andrea Minei, and Stewart Novick of Wesleyan University provided generous assistance with the microwave spectrometer. John Montgomery, Jr. provided assistance with the computations and scaling. NSF provided funds for construction of the spectrometer.

\section{References}

1. Bunn, C. W.; Howells, E. R. Nature 1954, 174, 549.

2. Shimanouchi, T.; Mizushima, S. J. Chem. Phys. 1955, 23, 707.

3. Iwasaki, M. J. Polym. Sci. 1963, A-1, 1099.

4. Sperati, C. A.; Starkweather, H. W., Jr. Fortschr. Hochpolym. Forsch. 1961, 2, 465.

5. Zhang, W. P.; Dorset, D. L. Macromolecules 1990, 23, 4322.

6. Dixon, D. A. J. Phys. Chem. 1992, 96, 3698.

7. Smith, G. D.; Jaffe, R. L.; Yoon, D. Y. Macromolecules 1994, 27, 3166.

8. Albinsson, B.; Michl, J. J. Phys. Chem. 1996, 100, 3418.

9. Röthlisberger, U.; Laasonen, K.; Klein, M. L.; Sprik, M. J. Chem. Phys. 1996, 104, 3692.

10. Watkins, E. K.; Jorgensen, W. L. J. Phys. Chem. A 2001, 105, 4118.

11. Borodin, O.; Smith, G. D.; Bedrov, D. J. Phys. Chem. B 2002, 106, 9912.

12. Ignatieva, L. N.; Beloiptsev, A. Y.; Kozlova, S. G. J. Struct. Chem. 2004, 45, 599.

13. D’Amore, M.; Talarico, G.; Barone, V. J. Am. Chem. Soc. 2006, 128, 1099.

14. Golden, W. G.; Brown, E. M.; Solem, S. E.; Zoellner, R. W. J. Mol. Spectrosc. 2008, 867, 22.

15. Fournier, J. A.; Bohn, R. K.; Montgomery, J. A., Jr.; Onda, M. J. Phys. Chem. A 2010, 114, 1118.

16. Munrow, M. R.; Subramanian, R.; Minei, A. J.; Antic, D.; MacLeod, M. K.; Michl, J.; Crespo, R.; Piqueras, M. C.; Izuha, M; Ito, T.; Tatamitani, Y.; Yamanou, K.; Ogata, T.; Novick, S. E. J. Mol. Spectrosc. 2007, 242, 129.

17. Ruoff, R.; Klots, T.; Emilsson, T.; Gutowsky, H. J. Phys. Chem. 1990, 93, 3142.

18. Gaussian 03, Revision B.05, Pople, J. A. and coworkers, Gaussian, Inc., Pittsburgh PA, 2003.

19. Balle, T. J.; Flygare, W. H. Rev. Sci. Instr. 1981, 52, 33.

20. Hight-Walker, A. R.; Lou, Q.; Bohn, R. K.; Novick, S. E. J. Molec. Struct. 1995, 346, 187.

21. Watson, J. K. G. In Vibrational Spectra and Structure, Vol. 6, Durig, J. R., Ed., Elsevier: Amsterdam, 1977, pp 1-89. 